\title{
The Influence of Nursing Intervention on Diabetic Patients with High Blood Pressure
}

\author{
Yun $\mathbf{W u}^{*}$ \\ Department of Endocrinology, The Third Affiliated Hospital of Guiyang Medical College, Guiyang, Guizhou 558000, China
}

\begin{abstract}
Objective: To observe the clinical symptoms of diabetes patients with high blood pressure, highlighting the influence and application value of nursing intervention on diabetic patients with hypertension. Methods: The data of 80 cases of diabetic patients with high blood pressure in our hospital from January 2014 to December 2014 were retrospectively analyzed. The patients were randomly divided into the experiment group and the control group. There were 40 patients of each group. Both patient groups were treated by the same means, subsequently, the control group were given routine nursing, while the experimental group were given programmed nursing to conduct care intervention. Finally, both clinical nursing effects were compared. Results: The numbers of patients with curative effect evaluation in the experimental group were marked higher than the control group patients. In terms of nursing satisfaction, the number of satisfied patients was larger than the control group, and the difference of the two groups was statistical significance $(p<0.05)$. Conclusion: Nursing intervention can strengthen the therapeutic effect of drugs, help to improve the cognitive ability of diabetic patients clinically which also improves the quality of life of the patients.
\end{abstract}

\section{KEYWORDS}

Diabetes combining hypertension

Nursing intervention

Application value

\section{Introduction}

Despite the advances in science and technology in China and improvement of living standards, the number of diabetes mellitus (DM) patients has been constantly rising. The increasing diabetes commonness has been a serious threat to human health and has become a worldwide public health problem. Studies have shown that the frequency of hypertension cases for people with diabetes were significantly higher than people without diabetes. More than half of the patients with chronic diabetes have hypertension. Hypertension will increase the development of cerebrovascular disease. The data showed that about $50 \%$ of patients with diabetes can also suffer from high blood pressure simultaneously. The early and long-term individualized clinical treatment should be emphasized in treating diabetes

Copyright $\odot 2015$ Yun Wu

doi: $10.18686 /$ jn.v4i3.6

Received: June 23, 2015; Accepted: August 12, 2015; Published online: August 22, 2015

This is an open-access article distributed under the terms of the Creative Commons Attribution Unported License (http://creativecommons.org/ licenses/by-nc/4.0/), which permits unrestricted use, distribution, and reproduction in any medium, provided the original work is properly cited.

${ }^{*}$ Corresponding author: Department of Endocrinology, The Third Affiliated Hospital of Guiyang Medical College, Guiyang, Guizhou 558000, China.E-mail:wuyun123y@sina.com and it is expected that the intervention of clinical nursing in the process of treatment is crucial. In order to explore the influence and application value of nursing intervention on diabetic patients with hypertension, the data of 80 cases of diabetic patients with high blood pressure in the hospital from January 2014 to December 2014 was retrospectively analyzed. The analysis was reported as below.

\section{The Data and Methods}

\subsection{General Information}

The data of 80 cases of diabetic patients with high blood pressure in our hospital from January 2014 to December 2014 was retrospectively analyzed. These patients were randomly divided into two groups, i.e. experimental and control group. There were 40 patients in each group and the patients met the WHO diagnostic criteria for diabetes in 1999. The patients consist of 44 male patients, 36 female patients, the ages of the patients were between 65 and 82, and the average age was 73 years old. All selected patients were diagnosed with type 2 diabetes mellitus with hypertension. The patients with severe liver disease, renal insufficiency, blood system diseases, severe myocardial disease, malignant tumor and other chronic disease were rejected. The selected patients are willing to cooperate to measure and record the blood sugar, blood pressure, and higher be- 
havior of obeying principle. The data of gender, age and illness were analyzed statistically with statistical significance $(p>0.05)$.

\subsection{The Clinical Nursing Intervention 2.2.1. The Development of Intervention Strategy}

The main content, implementation steps of the intervention time and frequency with the reference to literatures related clinical nursing of diabetic patients with hypertension, were determined by a research group made up of the endocrinology physicians, cardiovascular physician and clinical nurses to strengthen the intervention methods of the clinical nursing intervention.

Clinical nursing intervention education involves diabetes education, diet education, exercise intervention and medication education module. The process of intervention was completed by clinical nurses through 4-times visit of patients, once every two weeks, through community health education and demonstration, blood pressure and blood glucose detection procedures. Strengthening intervention was completed via phone calls after a week of intervention or between intervals of the two interventions, respectively.

\subsubsection{Main Content of Intervention}

Diabetes education: To make the patients and family understand and master diabetes prevention measures, medical staff should adopt multi-channel way to ensure the permeability of related knowledge to the patients and effectively reduce the occurrence of complicated diseases by high blood pressure. In addition, the medical staff should also teach patients how to test their blood and urine sugar level themselves for them to be able to check the blood pressure regularly.

Guide for medicine: The elderly diabetes patients must accurately grasp the period of medicine consumption and explain the half-life duration of drugs to avoid the peak of blood sugar levels (insulin secretion peak) to fall and result in low blood sugar. In addition, the health care workers must conduct strict supervision on the use of drugs among elderly diabetic patients, to avoid excessive use or re-usage of drugs and improper usage, which may lead to hypoglycemia.

Movement direction: Elderly patients with diabetes can directly reduce the blood glucose and enhance the role of insulin through exercise. However, they should move according to their strengths, gradually increase the amount and time of exercises according to the physical condition. They should also choose some relatively simple way of movement, and appropriately control the movement time, do not do extreme exercise.

Life coaching: When the patients were discharged, medical personnel should inform patients and their family members to bring along their own account, while reminding them to use the glucose meter to detect the blood sugar on time and adjusting the dosage according to the inspec- tion situation. Patients at home should follow the corresponding to medication and diet treatment principles, while the drug use was being monitored by family members. Patients also should regularly pay visit to the hospital for early prevention and treatment of a variety of complications, and subsequently improve the quality of life of the related patients.

\subsection{Curative Standard}

The performance evaluation includes knowledge evaluation and disease control success rate evaluation. The knowledge evaluation covers the blood pressure and blood sugar monitoring before and after the intervention. The knowledge evaluation was completed by using self-designed questionnaires. The questionnaire includes medical knowledge, scientific diet management, psychological counseling and debugging, reasonable exercise tutorials and life nursing knowledge. A total of five big modules, each module of the scoring range is from 0 to 20 points, covering four entries. Meanwhile, the success rate for disease control mainly includes the success rate for blood pressure and blood sugar control success rate.

Curative effect evaluation standard according to the guidelines of the China hypertension prevention for control standard were divided into:

Effective: Diastolic pressure drop is less than $10 \mathrm{mmHg}$, but blood pressure returned to normal levels. Invalid: Symptoms did not get better upon treatment or the results on the satisfaction survey from nursing care treatment effect indicated satisfaction, general or not satisfied.

\subsection{Statistical Processing Method}

All data were processed using SPSS 10.0 statistical software for statistical analysis, measurement data was represented by average number \pm standard deviation $(x \pm s)$, using $t$ to test, counting data adopting test $(p<0.05)$ showed that difference had statistical significance. The results of the data of 80 cases of diabetic patients with high blood pressure in our hospital were retrospectively analyzed. The patients were randomly divided into two groups. The control group was treated by routine nursing methods while the experimental group was treated by programmed nursing care intervention. By comparison of two groups of patients with clinical nursing effect, the details are shown in Tables 1 and 2.

Table 1. The comparison of clinical efficacy for two groups of patients.

\begin{tabular}{ccccc}
$\begin{array}{c}\text { Group } \\
\text { type }\end{array}$ & $\begin{array}{c}\text { Case } \\
\text { number }\end{array}$ & $\begin{array}{c}\text { Markly } \\
\text { effective }\end{array}$ & Effective & $\begin{array}{c}\text { Not } \\
\text { effective }\end{array}$ \\
\hline $\begin{array}{c}\text { The experiment } \\
\text { group }\end{array}$ & 40 & 29 & 10 & 1 \\
$\begin{array}{c}\text { The control group } \\
X^{2}\end{array}$ & 40 & 25 & 9 & 6 \\
$p$ value & $/$ & 10.1222 & 8.786 & 9.471 \\
\hline
\end{tabular}


Table 2. The comparison of satisfaction for the two groups.

\begin{tabular}{ccccc}
\hline $\begin{array}{c}\text { Group } \\
\text { type }\end{array}$ & $\begin{array}{c}\text { Case } \\
\text { number }\end{array}$ & Satisfaction & $\begin{array}{c}\text { Moderate } \\
\text { satisfaction }\end{array}$ & $\begin{array}{c}\text { No } \\
\text { satisfaction }\end{array}$ \\
\hline $\begin{array}{c}\text { The } \\
\text { experiment } \\
\text { group }\end{array}$ & 40 & 28 & 9 & 3 \\
\hline $\begin{array}{c}\text { The } \\
\text { control } \\
\text { group }\end{array}$ & 40 & 25 & 5 & 10 \\
\hline$X^{2}$ & $/$ & 8.182 & 6.402 & 8.065 \\
\hline$p$ value & $/$ & 0.0043 & 0.009 & 0.0012 \\
\hline
\end{tabular}

Based on the above tables, we can clearly know that the number of patients evaluated with curative effect and satisfied with nursing was markedly higher in experimental group as compared to control group. Both groups had significant difference $(p<0.05)$.

\section{Discussion}

Diabetes and high blood pressure is the most prominent factors which cause large blood vessels and capillaries pathological changes. Combined presence of diabetes and hypertension increases the risk of heart, brain and kidney damage of patients. Thus, it can be said that it is vital to pay attention to clinical treatment and nursing for diabetic patients with high blood pressure. The patients also need to realize that apart from the aid of drug, the treatment of diabetes with hypertension also involves the self apprehension of patients to adapt healthy living habits and be able to treat the disease in the most proper manner. Since diabetes and high blood pressure are chronic diseases, basic knowledge over the matter is important to guarantee for chronic disease self-management and control. We used the knowledge of diabetes and high blood pressure as the main content of clinical nursing intervention. The intervention covers diabetes education, diet, exercise and medication treatment education. The effect of intervention was strengthened by periodic phone calls. Nursing intervention can also educate the patients on hypertension and diabetes which directly improves the confidence of patients in the process of treatment and subsequently achieve a good therapeutic effect. With the continuous development in modern nursing, the nursing staff did not only engage in nursing technical service as subjected in the past. In fact, the nursing method has gradually begun to pay more attention to the patient's mental state to improve the quality of life of the patients, in comparison to the attention paid on the disease itself.
It is also expected that the scientific and comprehensive method of nursing can improve the cognition of the disease, easing of patients' depression, enhancing the confidence of patients to treat disease while helping the patients to correctly grasp the calculation of daily calorie taking from food and proper amount of exercise. The development of reasonable eating habits in addition of psychological nursing and health education do not only help patients to strengthen the drug treatment effect, it also improves the patients' life quality but also can prevent patients from some complication after treatment.

In summary, correct and reasonable nursing intervention can strengthen the result of clinical treatment of diabetic hypertension patients, helping patients to develop healthy living habits, also effectively prevent and control the occurrence of complications. It is also important to assure and regulate the patient's psychological counsellingand prepare related nursing for patients so that the patients can overcome the ailment with a positive, healthy, sunshine and optimistic attitude. Active treatment can also promote to the development of the disease treatment.

\section{Reference}

1. Wei HJ, Cheng F. Clinical characteristics and related factors analysis of chronic complications of patients with type 2 diabetes. Journal of Hebei Medicine. 2012;33(18):2742-2743.

2. Liu D, Shen J, Huang $X$, et al. The life quality analysis of influencing factors for diabetic patients. Contemporary Nurse. 2012;(3):4-6.

3. Dai $\mathrm{H}$, Liu $\mathrm{B}$. The pathogenesis and treatment progress of diabetes hypertension. Chinese Journal of Diabetes. 2013;18(11):870-871.

4. Lin T, Lin J, Chen $\mathrm{H}$. The influence of life quality of comprehensive nursing intervention for patients with type 2 diabetes mellitus and hypertension. Journal of Modern Hospital. 2012;10:64-66.

5. Feng J. The influence of Telmisartan on blood fat and insulin resistance for patients with diabetic hypertension. Modern Medicine. 2012;17:127-128.

6. Huang L, Fu HF. The influence of comprehensive nursing intervention on quality of life for patients with type 2 diabetes mellitus and hypertension. Journal of Qiqihar Medical College. 2012;19:2694-2695.

7. Qin Y. The influence of continuity nursing intervention on the quality of life for elderly patients with type 2 diabetes mellitus and hypertension. Journal of Medical Theory and Practice. 2015;7:973-975. 